\title{
Structural Analysis of the Shigella Virus Sf14 Capsid
}

\author{
Sarah Doore, Sundharraman Subramanian and Kristin Parent
}

Michigan State University, East Lansing, Michigan, United States

Viruses are minimally comprised of a genome that is protected from the environment by a protein coat. Many viruses are spherical, with the coat proteins forming an icosahedral capsid around the genome. The T number, or triangulation number, of a capsid describes the geometry of this icosahedral shell [1]. Of the bacterial viruses - known as bacteriophages or phages - most have icosahedral capsids that display $\mathrm{T}=7$ or T=13 symmetry, which have been analyzed in terms of assembly, structure, and stability [2].

Recently, several members of the Mooglevirus group of phages were isolated from environmental sources in Michigan and Nebraska [3,4]. These were most frequently isolated using the bacterium Shigella flexneri. Across two years of sampling, 16 Mooglevirus-like S. flexneri phages were found. By contrast, none were isolated on Salmonella and only one was found on E. coli. Mooglevirus characteristics include an icosahedral head with a long, contractile tail that attaches to the host. These newly isolated Mooglevirus-like phages have an uncommon genome size of 85.0 - 95.0 kilo base pairs, suggesting they may have similarly uncommon capsid geometry. An initial low-resolution structure (15 ̊) revealed these phages' capsids exhibit $\mathrm{T}=9$ icosahedral symmetry. From hundreds of available virus structures, only three-including one of these environmental isolates - exhibit $\mathrm{T}=9$ icosahedral symmetry. The other two $\mathrm{T}=9$ capsids are resolved to $14 \AA$ (EMD-1472) and $18 \AA$ (EMD-6043) [5,6].

Using cryo-electron microscopy, we have since obtained a $4.5 \AA$ structure of the Mooglevirus-like Sf14 virion. To our knowledge, this is the first high-resolution structure of a $\mathrm{T}=9$ virus. On the virion, decoration-like proteins bind preferentially to only the hexamers surrounding the pentamers at each icosahedral vertex. These decoration proteins have two immunoglobulin-like domains, which are similar to the single immunoglobulin domain in the $\mathrm{T}=13$ bacteriophage $\mathrm{T} 5$ [7]. However, in T5, the decoration protein binds to the center of all hexamers of the capsid. The mechanism conferring hexamer-specific binding in Sf14 has yet to be determined. In addition, the function of this protein is currently unknown but is hypothesized to play a role in capsid stability. Determining the structure and measuring the stability of capsids with or without decoration proteins may indicate how and why these proteins are associated with the virion.

\section{References}

[1] Caspar DL and Klug A, CSH Sym Quant Bio 27 (1962), 1.

[2] Carrillo-Tripp M, et al, Nucleic Acids Res 37 (2009), D436.

[3] Doore SM, et al, J. Virol. 92 (2018), e02117-17.

[4] Doore SM, et al, Virology 538 (2019), 45.

[5] Choi KH, et al, J Mol Biol 378 (2008), 726.

[6] Grose JH, et al, J Virol 88 (2014), 11846.

[7] Vernhes E, et al, Sci Rep 7 (2017), 41662. 